\title{
Correção endovascular de aneurisma de aorta abdominal em paciente com rim em ferradura: relato de caso e revisão das estratégias terapêuticas
}

\author{
Endovascular aproach of abdominal aortic aneurysm in a patient with a horseshoe kidney: case \\ report and review of therapeutic strategies
}

Reparación endovascular de aneurisma de aorta abdominal en paciente con riñón en herradura: reporte de caso y revisión de estrategias terapéuticas

\section{Resumo}

O rim em ferradura é uma das anomalias urológicas congênitas mais comuns e está presente em cerca de $0,12 \%$ dos pacientes com aneurisma de aorta abdominal. A causa mais comum é a degeneração da parede pela doença aterosclerótica, e, entre outras causas, incluem-se infecção, necrose cística, artrite, trauma e doenças do tecido conjuntivo. O reparo cirúrgico convencional está associado a dificuldades técnicas que provavelmente aumentam a morbidade e a mortalidade, mas que podem ser evitadas com o tratamento endovascular. A correção do aneurisma de aorta abdominal via endovascular é uma técnica menos invasiva se comparada a técnica aberta convencional, onde é feito um reforço da parede arterial para evitar que o seguimento afetado se rompa. No presente artigo. relatamos um caso de um paciente do sexo masculino de 69 anos com rim em ferradura e aneurisma de aorta abdominal infrarrenal, que foi submetido ao reparo endovascular do aneurisma com sucesso.

Palavras-chave: Aneurisma de aorta abdominal; Anormalidade congênita; Implante de prótese vascular.

\begin{abstract}
Horseshoe kidney is one of the most common congenital urologic anomalies and is present in approximately $0.12 \%$ of patients with abdominal aortic aneurysm. The most common cause is wall degeneration from atherosclerotic disease, and other causes include infection, cystic necrosis, arthritis, trauma, and connective tissue diseases. Conventional surgical repair is associated with technical difficulties that probably increase morbidity and mortality, but that can be avoided with endovascular treatment. Endovascular abdominal aortic aneurysm repair is a less invasive technique compared to the conventional open technique, in which the arterial wall is reinforced to prevent the affected segment from breaking. In this article. we report the case of a 69-year-old male patient with horseshoe kidney and infrarenal abdominal aortic aneurysm, who underwent successful endovascular aneurysm repair.
\end{abstract}

Keywords: Abdominal aortic aneurysm; Congenital abnormalities; Blood vessel prosthesis implantation. 


\section{Resumen}

El riñón en herradura es una de las anomalías urológicas congénitas más frecuentes y está presente en aproximadamente el $0,12 \%$ de los pacientes con aneurisma de la aorta abdominal. La causa más común es la degeneración de la pared por enfermedad aterosclerótica, y otras causas incluyen infecciones, necrosis quística, artritis, traumatismos y enfermedades del tejido conectivo. La reparación quirúrgica convencional se asocia a dificultades técnicas que probablemente aumentan la morbilidad y la mortalidad, pero que pueden evitarse con tratamiento endovascular. La reparación endovascular del aneurisma de la aorta abdominal es una técnica menos invasiva en comparación con la técnica abierta convencional, en la que se refuerza la pared arterial para evitar la rotura del segmento afectado. En este articulo. Presentamos el caso de un paciente masculino de 69 años con un riñón en herradura y un aneurisma de la aorta abdominal infrarrenal, que fue sometido con éxito a la reparación endovascular de un aneurisma.

Palabras clave: Aneurisma de aorta abdomina; Anomalías congénitas; Implante de próteses vascular.

\section{Introdução}

A doença aneurismática é conhecida desde a antiguidade, onde foi descrita como "uma dilatação localizada com pulsação que nos casos de ferida sangraria abundantemente". (Cruz, 2012)

A dilatação aneurismática da aorta ocorre quando seu diâmetro excede $50 \%$ do previsto como normalidade para aquela idade e superfície corporal. (Albuquerque et al., 2009). Ë resultante geralmente de uma degeneração da túnica média arterial que promove um aumento lento, porém contínuo do diâmetro do vaso. Outras afecções podem estar associadas a gênese do aneurisma da aorta abdominal (AAA), como infecção, necrose cística da túnica média, artrite, trauma ou mesmo doenças do tecido conjuntivo. (Ministério da Saúde, 2017).

Os aneurismas aórticos abdominais são mais comuns nos homens do que nas mulheres (aproximadamente 8:1). Homens brancos têm uma prevalência mais alta e há incremento do risco em desenvolver com a idade, particularmente após os 70 anos. O tabagismo é o fator de risco mais importante. Ocorrem em 10\% nos homens com hipertensão, doenças arteriais periférica, carotídeas ou coronárias. Existe correlação com a história familiar, ou seja, parentes de primeiro grau de pacientes com aneurisma aórtico abdominal apresentam maior predisposição à doença. (Cruz, 2012)

A localização mais comum dos aneurismas aórticos é na aorta abdominal, e mais de $40 \%$ dos pacientes com aneurismas infra-renais podem apresentar aneurisma em outro sítio. A incidência de AAA é de 21 em 100.000 pacientes-ano. (Cruz, 2012) Por outro lado, a associação de rim em ferradura com aneurisma de aorta abdominal (AAA) é rara, estando presente em apenas $0,12 \%$ dos pacientes que passam por correção cirúrgica, aberta ou endovascular, dos AAA. (Coelho et al., 2020).

A correção do aneurisma de aorta abdominal via endovascular é uma técnica menos invasiva se comparada a técnica aberta convencional, onde é feito um reforço da parede arterial para evitar que o seguimento afetado se rompa. O procedimento consiste em realizar punção ou dissecção da artéria femoral na região do trígono femoral, visualização do aneurisma por método de imagem arteriográfica e liberação da endoprótese no interior da aorta, excluindo o sítio aneurismático. (Antunes et al., 2018).

No processo de migração dos rins para a fossa renal, eles cruzam as artérias umbilicais. Caso ocorra alteração na posição destes vasos podem promover a fusão dos blastemas nefrogênicos (Maranhão et al., 2013), ocasionando alterações na morfologia dos rins, a exemplo, do rim em ferradura ou rim em panqueca. O rim em ferradura é uma das anomalias congênitas mais prevalentes. A sua incidência está estimada em um a cada 600 a 800 indivíduos. (Silvestre et al., 2013).

A correção do AAA associado a rim em ferradura envolve alguns problemas. Primeiramente, a presença do parênquima renal, que pode dificultar a exposição do aneurisma. E finalmente, o desafio técnico de corrigir o aneurisma quando dele emergem artérias anômalas importantes para o suprimento vascular renal. Portanto, considerando as particularidades anatômicas entre o aneurisma e a alteração renal, a cirurgia convencional pode se tornar um grande desafio. 
$\mathrm{Na}$ atualidade, a terapêutica endovascular tem sido recomendada como importante alternativa e preferencial na maioria dos casos de malformações renais congênitas. (Coelho et al., 2020).

O presente artigo tem como propósito o relato de caso de um paciente de 69 anos, com rim em ferradura, aneurisma de aorta abdominal sintomático, disfunção renal leve e doença coronariana multiarterial. Foi realizado tratamento endovascular com implante de endoprótese e discutido as estratégias terapêuticas.

\section{Metodologia}

Trabalho realizado com metodologia de estudo de caso de característica qualitativa e descritiva. De acordo com Pereira et al., 2018, pesquisas com esta finalidade caracterizam-se por trazer detalhamento científico sobre um determinado assunto e estudá-lo acuradamente. A descrição do estudo apresentado é realizada considerando o melhor protocolo terapêutico disponível e a cronologia do caso em questão. Assim, feito relato de caso e revisão de literatura com base em um paciente coronariopata portador de aneurisma de aorta abdominal sintomático associado a rim em ferradura, sendo proposto a partir destes achados o tratamento mais indicado.

Respeitando os aspectos éticos, legais e estando em concordância com a Declaração de Helsinque e a Resolução 466/12 do Conselho Nacional de Saúde (CNS). O participante do estudo foi incluído após assinatura no Termo de Consentimento Livre e Esclarecido (TCLE) declarando aceitar participação no estudo e liberando o uso de sua imagem para fins acadêmicos/científicos.

\section{Resultados}

Paciente do sexo masculino, 69 anos, portador de aneurisma de aorta abdominal infrarrenal e com antecedentes mórbidos pessoais de hipertensão arterial sistêmica, dislipidemia e tabagismo. Proveniente do Hospital Pronto Socorro Mário Pinotti (HPSM). Informou que o aneurisma foi identificado durante realização de ultrassonografia a para investigação de outras queixas.

Ao exame físico, apresentava-se normotenso e o exame abdominal evidenciava massa pulsátil em topografia de epigástrio. Apresentava todos os pulsos amplos e simétricos bilateralmente. Os exames laboratoriais, apresentavam discreta anemia, glicemia normal e creatinina alterada (clerance de $35 \mathrm{~mL} / \mathrm{min} / 1,73 \mathrm{~m}^{2}$ ).

Foi diagnosticado durante os exames pré operatórios, com doença cardíaca isquêmica multiarterial. Realizou angiotomografia computadorizada de tórax e abdome que evidenciou AAA, fusiforme, infrarrenal, iniciando aproximadamente 20mm após a emegencia da artéria renal esquerda e estendendo-se até a bifurcação aórtica, com diâmetro transverso máximo de $7,9 \mathrm{~cm}$ e com envolvimento de artéria ilíaca comum esquerda que apresentava dilatação de 3,4cm (Figuras 1 e 2). Achado incidental extravascular de rim em ferradura em íntimo contato com o aneurisma. $\mathrm{O}$ istmo constituído por parênquima renal normal nutrido por ramo aórtico acessório e uma artéria renal principal para cada um dos rins. 
Figura 1. Aneurisma de aorta abdominal e presença de rim em ferradura.

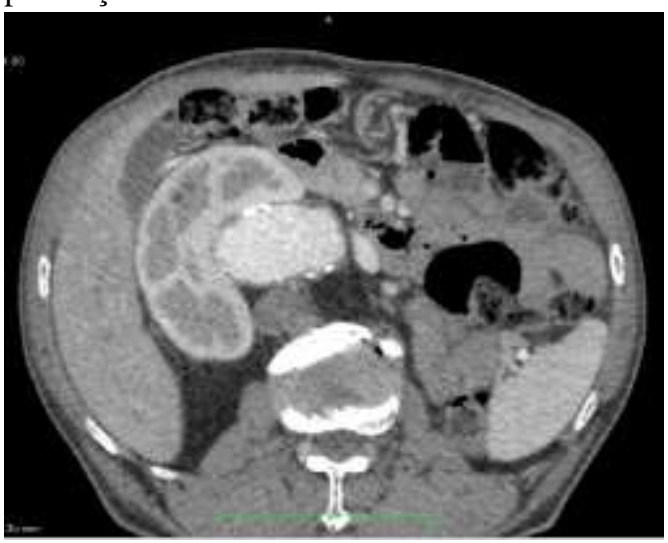

Fonte: Autores.
Figura 2. Aneurisma de aorta abdominal $7.9 \mathrm{~cm}$ e presença de rim em ferradura.

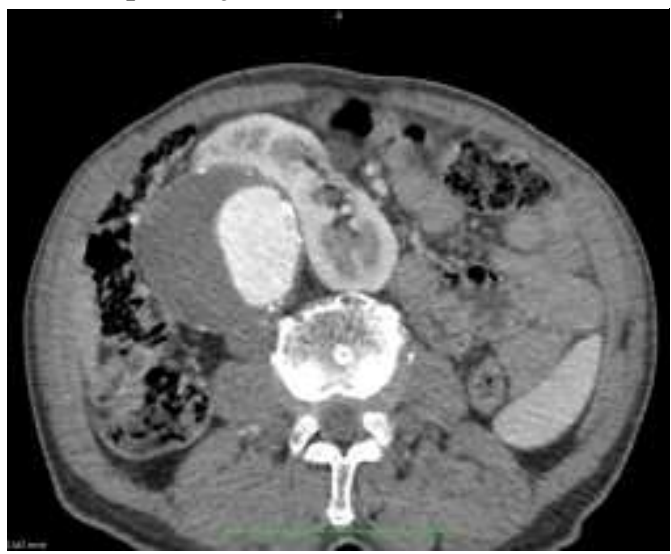

Fonte: Autores.

Após estudo clínico e avaliações de time multiprofissional o paciente foi classificado como risco cirúrgico grau III (segundo classificação de Golfmann) e risco anestésico (ASA) III.

Em virtude do acometimento coronariano a equipe de cirúrgica cardíaca da instituição realizou a revascularização com uma ponte de mamária e 02 pontes de veia safena magna. O paciente evoluiu de forma satisfatória recebendo alta da UTI no terceiro dia de pós operatório e foi liberado para correção de aneurisma após 3 semanas.

Após avaliação e discussão da equipe de cirurgia vascular e cardiologia e baseado na anatomia favorável, foi optado por abordagem endovascular.

O procedimento foi realizado sob anestesia geral e com acesso femoral oblíquo bilateral, com uma endoprótese Braile ${ }^{\circledR}$ bifurcada com ancoragem distal em artérias ilíacas comun (corpo principal - LINUS 30x14/155/75 20F, 14x22/120 16F e 14/140 - 16F). Durante a intervenção endovascular foi observado a íntima relação do aneurisma aórtico com o rim em ferradura, porém com colo proximal satisfatório pra ancoramento proximal da endoprótese (Figura 3). Houve um agravamento da função renal nos primeiros dias de pós-operatório retornando ao nível basal após o terceiro dia de PO. Recebeu alta da UTI para a enfermaria $48 \mathrm{~h}$ após o procedimento e alta hospitalar no $7^{\circ}$ dia de $\mathrm{PO}$.

Figura 3. Angiografia de controle evidenciando vasos viscerais e colo proximal

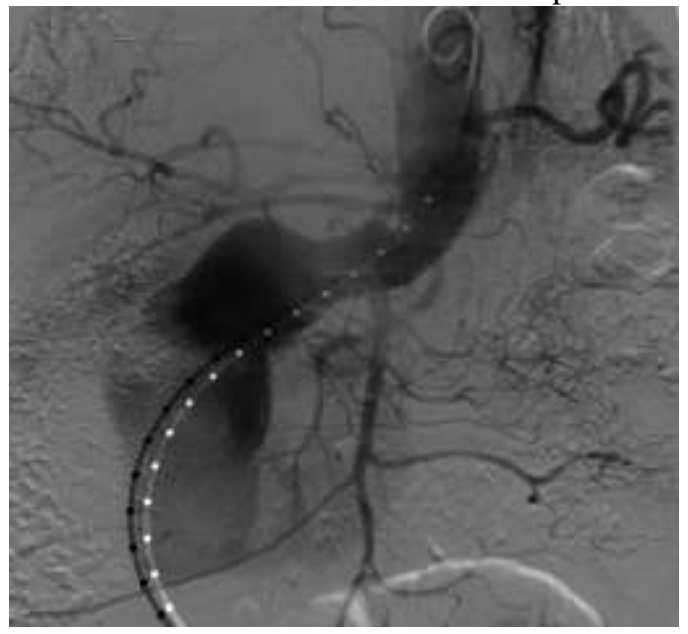

Fonte: Autores

Os exames angiotomográficos de seguimento evidenciaram endoprótese com ótima implantação, adequada coaptação 
e sem sinal de endoleaks. Na região do istmo, observou-se pequena área de infarto renal correspondente a aproximadamente $20 \%$ do parênquima renal total. Estudos tomográfico de controle após 12 meses de seguimento revelou redução progressiva do aneurisma e sem sinais de endoleaks (Figuras 4 e 5). Durante todo o período de seguimento, o paciente permaneceu assintomático, com bom controle dos níveis tensionais e com exames laboratoriais sem alterações significativas.

Figura 4. Vista posterior da Angiotomografia de controle 12 meses após tratamento.

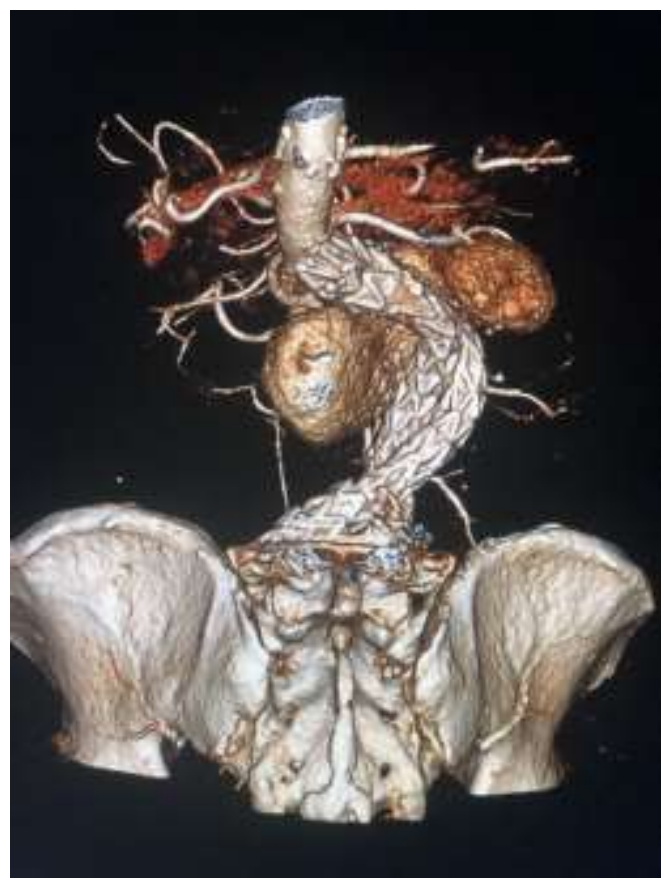

Fonte: Autores.
Figura 3. Vista antero-lateral da Angiotomografia de controle 12 meses após tratamento.

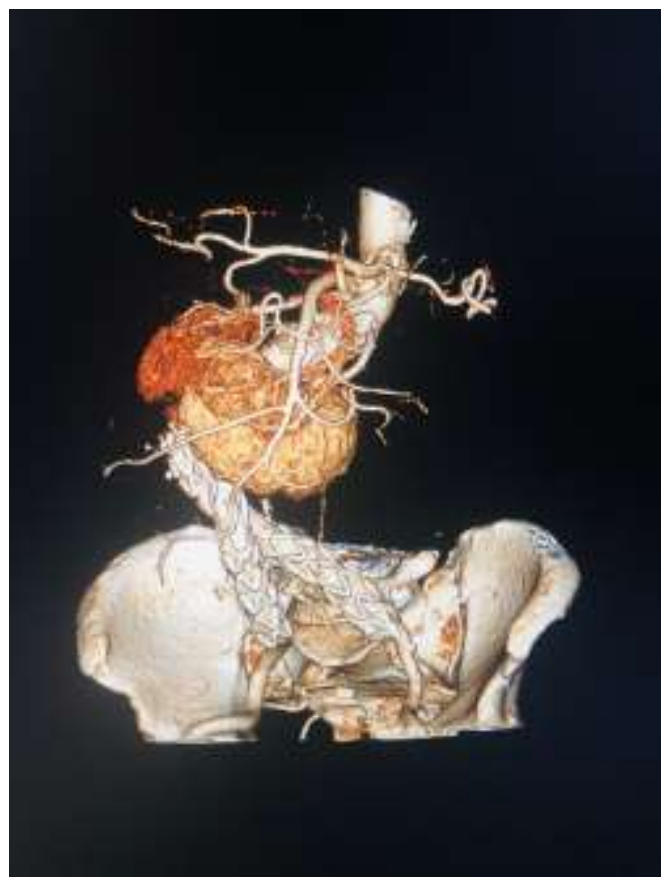

Fonte: Autores.

\section{Discussão}

O primeiro caso, bem sucedido, de tratamento de um AAA em paciente com rim em ferradura foi descrito por Phelan et al em 1957. (Coelho et al., 2020). Já em 1997, Ferko et al descreveram o primeiro caso da associação de um AAA e rim em ferradura corrigido pela técnica endovascular. (Antunes et al., 2014).

Algumas possibilidades terapêuticas são levantadas para pacientes portadores desse duplo acometimento (AAA e rim em ferradura): tratamento cirúrgico aberto com acesso transperitoneal, tratamento cirúrgico aberto com acesso retroperitoneal ou tratamento endovascular. (Fabiani et al., 2019).

A correção por técnica aberta convencional possibilita, por acesso transperitoneal, melhor exposição do aneurisma e do rim, porém a presença do istmo renal prejudica a exposição adequada do AAA (Davidovic et al., 2018). Já a abordagem retroperitoneal tem a vantagem de evitar a manipulação do istmo e do trato urinário; no entanto, o acesso à artéria ilíaca comum direita distal (e de sua bifurcação) torna-se limitado. Desta forma, o emprego da terapêutica por via endovascular tem a vantagem de evitar a dificuldade da exposição cirúrgica da aorta e do próprio rim em ferradura que a recobre. Mas, nesta modalidade há maior risco de infartos renais pós-operatórios, uma vez que a vascularização do rim em ferradura é bastante variável e, por vezes, artérias renais acessórias originadas do AAA são responsáveis pela irrigação de quantidade não desprezível de parênquima renal. (Coelho et al., 2020).

Assim, desde a primeira descrição por Juan Carlos Parodi (Saadi, 2009), a técnica de correção endovascular do AAA tem evoluindo e proporcionado múltiplas possibilidades de tratamento mesmo em casos complexos em associação com mal 
formações em outros sistemas, tendo em vista o desenvolvimento de materiais de menor perfil, resistentes e flexíveis e com melhores mecanismos de fixação. (Saadi, 2009).

A anatomia do paciente também é fundamental para o sucesso do procedimento, seja ele convencional ou endovascular, e a complexidade anatômica implica em algumas particularidades, independente da técnica cirúrgica adotada. (Bounssir et al., 2020).

O rim em ferradura, apresenta variações anatômicas arteriais e venosas, assim como do parênquima renal e das vias excretoras, o que motiva a dificuldade técnica ou mesmo impossibilita a abordagem convencional (Coelho et al., 2020). Na maioria dos casos, o ponto de fusão ocorre entre os polos inferiores dos rins, que se encontram mais próximos da linha média do que os rins normais. $\mathrm{O}$ ístmo, situado mais frequentemente na frente da aorta ou da veia cava inferior, une as duas massas renais e pode conter parênquima funcionante ou corresponder a uma faixa de tecido fibroso, por isso pode ser necessária à sua avaliação funcional por meio de radionuclídeo antes de qualquer procedimento intervencionista. O próprio istmo dificulta a rotação renal, assim como sua ascensão devido à artéria mesentérica inferior. (Maranhão et al., 2013). Nos casos em que há parênquima funcionante no istmo, deve-se evitar a secção dele, pois, além de maior dificuldade técnica, há também maior risco de contaminação do campo e subsequente infecção da prótese, visto que, em alguns rins em ferradura, há a presença de infecção crônica. (Coelho et al., 2020).

São várias as classificações de rim em ferradura, a maioria de baseia nas variações de suprimento sanguíneo para o órgao. A descrita por Eisendrath et al. é a mais utilizada e subdivide os achados anatômicos em 5 tipos: Tipo I: uma artéria renal para cada lado do rim em ferradura. Corresponde a $20 \%$ dos casos; Tipo II: uma artéria renal para cada lado da aorta e um ramo aórtico para o istmo. Corresponde a 30\% dos casos; Tipo III: duas artérias para cada lado do rim em ferradura e um ramo aórtico para o istmo. Corresponde a 15\% dos casos; Tipo IV: duas artérias para cada lado do rim em ferradura associada a um ou mais ramos ascendentes ilíacos. Corresponde a 15\% dos casos; Tipo V: múltiplas artérias renais, com origem na aorta, nas mesentéricas e nas ilíacas. Corresponde a $20 \%$ dos casos (Saadi, 2009). Assim, o caso relatado melhor se enquadra no tipo II.

O objetivo primário de todo tratamento endovascular do AAA é a prevenção da ruptura e suas potenciais consequências. Outros propósitos incluem a diminuição gradativa do tamanho aneurismático e a ausência de vazamentos como marcadores de risco continuado de ruptura. (Mikam et al., 2021).

Desta forma o caso apresentado corresponde a um paciente de alto risco cirúrgico portador de rim em ferradura tipo II no qual, após avaliação das características anatômicas e critérios clínicos cardiológicos, se considerou elegível para correção endovascular do AAA. Apresentou evolução favorável e encontra-se em seguimento ambulatorial.

\section{Conclusão}

$\mathrm{O}$ tratamento endovascular do AAA associado ao rim em ferradura vem sendo globalmente abordado, não existindo ainda consenso na literatura quanto ao melhor tratamento. A opção no presente caso foi a correção endovascular do AAA em associação com rim em ferradura, mas a avaliação completa dos parâmetros clínicos e anatômicos devem ser considerados para a elegibilidade da técnica mais adequada e das possíveis repercussões na má formação urológica.

\section{Referências}

Albuquerque, L. C., Braile, D. M., Palma, J. H., Saadi, E. K., Almeida, R. M. S. de, Gomes, W. J., \& Buffolo, E. (2009). Diretrizes para o tratamento cirúrgico das doenças da aorta da Sociedade Brasileira de Cirurgia Cardiovascular: atualização 2009. Revista Brasileira de Cirurgia Cardiovascular, 24(2), 7-33. https://doi.org/10.1590/s0102-76382009000300004

Antunes, I., Machado, R., Teixeira, S., Rego, D., Ferreira, V., Golçalves, J., Teixeira, G., Veiga, C., \& de Almeida, R. (2018). Tratamento endovascular de aneurisma da aorta abdominal em doente com rim em ferradura. ACV Journal, 1(14), http://www.scielo.mec.pt/pdf/ang/v14n1/v14n1a14.pdf 
Bálint, S., Mihály, Z., Oláh, Z., \& Sótonyi, P. (2020). Nyitott hasi aortaaneurysma-műtétek patkóvese fennállása esetén. Orvosi Hetilap, 161(46), 1966-1971. https://doi.org/10.1556/650.2020.31863

Bounssir, A., Bakkali, T., Taghi, H., Sefiani, Y., \& Lekehal, B. (2020). Best strategy in managing the association of Horse-shoe-Kidney and Abdominal Aortic Aneurysm: Case report. International Journal of Surgery Case Reports, 75, 11-15. https://doi.org/10.1016/j.jscr.2020.08.034

Bowden, S., \& Roche-Nagle, G. (2021). Fenestrated endovascular abdominal aortic aneurysm repair with concomitant horseshoe kidney. BMJ Case Reports, 14(1), e236755. https://doi.org/10.1136/bcr-2020-236755

Coelho, G. M. A., França, R. A. A., Rocha, R. D. L. da, Pantoja, M. de O., Lacerda, P. de S., Souza, J. E. dos S., Bernardes, M. V., \& Cavalcante, L. P. (2020). Aneurisma de aorta abdominal sintomático associado a rim em ferradura. Jornal Vascular Brasileiro, 19. https://doi.org/10.1590/1677-5449.200088

Cruz, B., \& Gomes, O. (2012). Surgical Treatment of Aortic Aneurysms. Cardiovasc Sci Forum, 7(3), 9. http://docplayer.com.br/6558892-Volume-7-numero3-julho-setembro-2012.html\#show_full_text

Davidovic, L. B., Markovic, M., Kostic, D., Zlatanovic, P., Mutavdzic, P., \& Cvetic, V. (2018). Open repair of ruptured abdominal aortic aneurysm with associated horseshoe kidney. International Angiology, 37(6). https://doi.org/10.23736/s0392-9590.18.04039-7

Docplayer. 7(3). Docplayer.com.br. http://docplayer.com.br/6558892-Volume-7-numero-3-julho-setembro-2012.html\#show_full_text

Eisendrath, D. N., Phifer, F. M., \& Culver, H. B. (1925). Horseshoe Kidney. Annals of Surgery, 82(5), 735-764. https://doi.org/10.1097/00000658192511010-00009

Fabiani, M. A., González-Urquijo, M., Riambau, V., Vaquero Puerta, C., Mosquera Arochena, N. J., Varona Frolov, S., \& Maldonado, T. S. (2019). EVAR Approach for Abdominal Aortic Aneurysm with Horseshoe Kidney: A Multicenter Experience. Annals of Vascular Surgery, 58, $232-237$. https://doi.org/10.1016/j.avsg.2018.10.042

Gabriele, P., Emanuele, G., Grilli Cicilioni, C., Sara, S., \& Luciano, C. (2019). Ruptured Abdominal Aortic Aneurysm and Horseshoe Kidney: Isthmus Sectioning By Endo GIA. Vascular and Endovascular Surgery, 53(7), 599-601. https://doi.org/10.1177/1538574419859451

Maranhão, C. P. de, Miranda, C., Santos, C., Farias, L., \& Padilha, I. (2013). Anomalias congênitas do trato urinário superior: novas imagens das mesmas doenças. Radiol Bras, 46(1), https://docplayer.com.br/1993156-Anomalias-congenitas-do-trato-urinario-superior-novas-imagens-das-mesmas-doencas.html

Melmer, P. D., Patel, A., Biswas, S., \& Borowicz, M. R. (2020). Horseshoe Kidney Isthmus Infarction After Percutaneous Endovascular Aortic Aneurysm Repair. Cureus. https://doi.org/10.7759/cureus.7279

Mikami, T., Kamada, T., Uchiyama, H., Kuroda, Y., Harada, R., Naraoka, S., \& Kawaharada, N. (2021). Successful Endovascular Repair of an Abdominal Aortic Aneurysm in a Patient with a Horseshoe Kidney and Accessory Renal Arteries Using an Aortic Cuff to Prevent Type II Endoleak. Annals of Vascular Diseases, 14(1), 52-55. https://doi.org/10.3400/avd.cr.20-00099

Ministério da Saúde, Comissão Nacional de Incorporação de Tecnologias no SUS - CONITEC. (2017). Diretriz Brasileira para o tratamento do Aneurisma de Aorta Abdominal. Link: http://conitec.gov.br/images/Relatorios/2017/Relatorio_Diretriz_AneurismaAortaAbdominal_Recomendacao.pdf.

Novero, E. R., Metzger, P. B., Angelieri, F. M. R., Colli, M. B. de O., Moreira, S. M., Izukawa, N. M., Rossi, F. H., \& Kambara, A. M. (2012). Correção endovascular do aneurisma da aorta abdominal: análise dos resultados de único centro. Radiologia Brasileira, 45(1), 1-6. https://doi.org/10.1590/s010039842012000100003

Pereira A. S., et al. (2018). Metodologia da pesquisa científica. UFSM

Peters, D., Obmann, M., Song, B., Nikam, S., Ryer, E., \& Mariner, D. (2020). Fenestrated endovascular aneurysm repair with concomitant horseshoe kidney. Journal of Vascular Surgery Cases and Innovative Techniques, 6(1), 14-17. https://doi.org/10.1016/j.jvscit.2019.09.002

Radermecker, M. A., Van Damme, H., Kerzmann, A., Creemers, E., \& Limet, R. (2008). Association of abdominal aortic aneurysm, horseshoe kidneys, and left-sided inferior vena cava: Report of two cases. Journal of Vascular Surgery, 47(3), 645-648. https://doi.org/10.1016/j.jvs.2007.08.053

Saadi, E. K. (2009). Correção endovascular do AAA. Revista Brasileira de Cirurgia Cardiovascular, 24(2). https://doi.org/10.1590/s010276382009000300009

Silvestre, J. M. da S., Schimit, G. T. F., Sardinha, W. E., Silvestre, G. da S., Tenório, G. O. S., \& Trevisan, F. B. (2013). Tratamento endovascular de aneurisma de aorta abdominal em paciente com rim em ferradura: relato de caso. Jornal Vascular Brasileiro, 12(1), 62-67. https://doi.org/10.1590/s167754492013000100013 